\title{
Anexo 2. Recursos disponibles
}

\author{
MANUEL DE LA PRIDA C.* y TANIA VIZCARRA O.**
}

Se presenta una lista de documentos sobre tabaquismo acompañadas de su disponibilidad en internet.

\section{Guías Clínicas}

1.- Guías para el tratamiento del tabaquismo basado en la evidencia. 2010. Argentina. http://files.sld.cu/antitabaquica/files/2013/04/guia-tto-basada-en-evidencia.pdf 2.- The New Zealand Guidelines for Helping People to Stop Smoking 2014. http://www.health.govt.nz/publication/new-zealand-guidelines-helping- people-stop-smoking 3.- National Cessation Guidelines Treatobacconet. http://www.treatobacco.net/en/page_549.php 4.- Canadian Smoking Cessation Clinical Practice Guideline. 2012.

https://www.nicotinedependenceclinic.com/English/CANADAPTT/Guideline/Introduction.aspx 5.- Guías NICE para profesionales de la salud. ¿Cómo dejar de fumar durante el embarazo y después del parto? 2010.

www.nice.org.uk/guidance/PH26

6.- Guía Nacional para el Abordaje del Tabaquismo Uruguay 2009.

http://www.who.int/fctc/reporting/Annexfiveurug.

7.- Manejo diagnóstico y tratamiento del tabaquismo en la práctica clínica diaria 32. 2015. SEPAR. Sociedad Española de Neumología y Cirugía Torácica.

https://issuu.com/separ/docs/manual_32

8.- Programa de Salud Cardiovascular MINSAL Chile. Manual para el Equipo de salud. "La cesación del Consumo de tabaco"

http://buenaspracticasaps.cl/recursos/documentos-minsal-programa-salud-cardiovascular/

9.- Guías de tratamiento del tabaquismo - Agency for Healthcare. España.

http://www.ahrq.gov/sites/default/files/wysiwyg/professionals/clinicians-providers/guidelinesrecommendations/tobacco/clinicians/update/treating_tobacco_use08_sp.pdf

\section{II.- Cursos de capacitación "on line"}

1.- Curso: "Tabaco y Salud Pública: De la Teoría a la Practica". Curso virtual gratuito de la OPS.

http://www.paho.org/chi/index.php?option=com_content\&view=article\&id=446:disponible-aula-cvspcurso-virtual-tabaco-salud-publica-teoria-practica\&Itemid $=214$

2.- Curso "Control Mundial de Tabaco". Curso virtual gratuito de la Facultad de Salud Pública Bloomberg de la Universidad Johns Hopkins.

http://globaltobaccocontrol.org/es/online_training

3.- Introducción a la prevención, control y cesación del tabaquismo. MINSAL. Chile.

http://minsal.uvirtual.cl/siminsal/\#collapseOne1_94_cap_act

4.- OPS/OMS. Curso Virtual en Control del Tabaco - PAHO WHO. 2016 http://www.paho.org/hq/index. php?option $=$ com_content\&view $=$ article $\& i d=3417 \% 3 \mathrm{~A} 2010$-virtual-course-on-tobaccocontrol\&catid $=$ $1281 \% 3$ Aart\&Itemid $=2516 \&$ lang=es

* Dpto. Enfermedades Respiratorias Hospital Dr. Gustavo Fricke, Viña del Mar.

Programa Tabaquismo Hospital Dr. Gustavo Fricke, Viña del Mar.

** Programa ERA CESFAM Alberto Allende Jones Talagante. 
5.- Curso virtual para el tratamiento de la adicción al tabaco. Argentina.

http://www.msal.gob.ar/tabaco/index.php/home/4-destacados-slide/314-continua-curso-virtual-para-eltratamiento-de-la-adiccion-al-tabaco

6.- Plataforma de Capacitación a Distancia del Programa SUMAR. Los cursos ofrecidos en esta plataforma son gratuitos, abiertos y auto gestionados. Argentina.

http://www.capacitacionsumar.msal.gov.ar/

\section{III.- Recursos para los pacientes}

1.- Programa en línea para dejar de fumar .Universidad de California Libre de cigarro.

https://stopsmokingsf.org/en/pages/brief_description

2.- Curso para dejar de fumar. Castelar.

https://es-la.facebook.com/Cursodejardefumar.Castelar

20 recursos online para dejar de fumar por internet. Fundación del Corazón.

http://www.fundaciondelcorazon.com/corazon-facil/blog-impulso-vital/2008-20-recursosonline-paradejar-d

3.- Recursos gratuitos para dejar de fumar / Smokefree Español.

https://espanol.smokefree.gov/recursos-gratuitos

4.- Guía para el paciente. "Se puede dejar de fumar. Claves para conseguirlo". Comité Nacional para la Prevención del Tabaquismo" España.

http://www.cnpt.es/listado-recursos.asp?id_categoria=30

5.- Recursos para ayudarlos a dejar de fumar / Dejar de fumar.

https://espanol.smokefree.gov/

6.- Tabaquismo: Consejos para como dejar de fumar: Medline Plus.

https://medlineplus.gov/spanish/ency/article/001992.htm

7.- Guía para dejar de fumar. Asociación Española Contra el Cáncer.

https://www.uclm.es/servicios/prevencion/actividades/Tabaco/guia_AECC.pdf

\section{IV.- Políticas sobre tabaquismo}

1.- El Convenio Marco Para el Control del Tabaco: Mayo 2003.

http://www.who.int/gb/fctc/PDF/inb6/einb65.pdf

2.- El Convenio Marco de la OMS para el Control del Tabaco: Un instrumento jurídico al servicio de la salud pública mundial. Rev. Chil. Enferm. Respir. v.19 n.3 2003.

http://www.scielo.cl/scielo.php?script=sci_arttext\&pid=S0717-73482003000300001

3.- Ministerio de Salud, Unidad de Tabaco.

4.- Revista SEPAR Prevención del Tabaquismo 18-2; Abril/Junio 2016.

https://issuu.com/separ/docs/prev_tab_18-2

5.- The Tobacco Atlas. Datos para Chile. 2015.

http://www.tobaccoatlas.org/country-data/chile

6.- Alianza Mundial para el Convenio Marco.

http://www.fctc.org

7.- Bloomberg Global Initiative to Reduce Tobacco Use. Fondos concursables para el control del Tabaco.

http://www.tobaccocontrolgrants.org/

8.- Tabaquismo. La situación Latinoamericana. IECS, Instituto de efectividad clínica y sanitaria, 2014.

http://www.iecs.org.ar/tabaco/

9.- Centro de Documentación Chile libre de Tabaco.

http://www.chilelibredetabaco.cl/centro-de-documentacion/

Correspondencia a:

Dr. Manuel de La Prida.

Email: manueldelaprida@gmail.com 